Research Article

\title{
Effect of Fractal-Based Fiber Clustering on Tensile Properties of BFRP
}

\author{
Jingjing He $\mathbb{D}^{1},{ }^{1}$ Junping Shi $\left(\mathbb{D},{ }^{2}\right.$ Yong Zhang $\left(\mathbb{D},{ }^{1}\right.$ Yali Bi $\mathbb{D D}^{1}$ and Lihao Fan $\mathbb{I D}^{1}$ \\ ${ }^{1}$ Northwest Engineering Corporation Limited, Power China, Zhangba Road 18, Xi'an, Shanxi, China \\ ${ }^{2}$ School of Civil Engineering and Architecture, Xi'an University of Technology, Xi'an, Shanxi, China \\ Correspondence should be addressed to Jingjing He; hejing_86@126.com
}

Received 11 May 2021; Accepted 28 June 2021; Published 6 July 2021

Academic Editor: Tianyu Xie

Copyright (c) 2021 Jingjing He et al. This is an open access article distributed under the Creative Commons Attribution License, which permits unrestricted use, distribution, and reproduction in any medium, provided the original work is properly cited.

To explore the clustering phenomenon of discontinuous fibers in composite materials, this paper deduces the fiber uniform distribution coefficient and analytical expressions of fiber clustering content based on fractal theory and establishes a tensile strength prediction model of fiber/epoxy resin composite materials containing cluster fibers. With basalt fiber/epoxy resin composites (BFRP) as an example, this paper analyzes the tensile strength law of BFRP under fiber clustering effect. The results show that when the fiber volume fraction is constant, the tensile strength of the composite in the presence of agglomerated fibers is only related to the fractal dimension of the circumference and cross-sectional area of the inner fiber agglomerate. The calculated value of the composite tensile strength based on fractal theory is lower than the experimental value, but closer to the experimental value than the approximate method. The research conclusions can provide theoretical support for strength prediction of fiber/ epoxy resin composites.

\section{Introduction}

Basalt fiber reinforced epoxy resin composites (BFRP) can be formed by mixing basalt fiber into epoxy resin materials at a certain ratio. BFRP materials are widely used in civil engineering, water conservancy, and hydropower and energy engineering fields because of their excellent performance, mechanical properties, and durability. At present, the research on BFRP materials focuses on the mechanical properties of the materials and their components, which are mainly in the following aspects.

First, the performance and mechanism of BFRP materials are analyzed, aimed at improving their mechanical properties and durability and exploring their internal mechanism. For example, [1-4] used different methods to treat and modify the fiber surface and studied the influence of fiber surface modification on the tensile properties of BFRP. The results showed that the mechanical parameters, such as tensile strength and elastic modulus, were significantly improved after the modification. Shokrieh et al. [5, 6] studied the sulfuric acid corrosion resistance of BFRP, analyzed the damage mechanics characteristics under bending load and corrosive medium, and obtained the life curve of BFRP materials. Bulut et al. [7] studied the influence of graphene nanoparticles on the damping and vibration characteristics of BFRP. The results indicated that when the mass fraction of graphene nanoparticles was $0.1 \%$ and $0.2 \%$, the damping and vibration characteristics of samples were significantly improved. Chelliah et al. [8] studied the influence of titanium carbide on the wear resistance of BFRP. Experiment results showed that the mass loss rate of BFRP decreased significantly when titanium carbide was added to BFRP materials and that it reached the lowest when the content of titanium carbide was $2 \%$. Sampath [9] analyzed the influence of nano-fly ash filler on the mechanical and chemical corrosion resistance of BFRP. Experiment results demonstrated that nano-fly ash filler could improve the properties of BFRP to varying degrees. Kim et al. [10] modified the fracture properties of BFRP with different concentrations of natural graphite sheets. The results suggested that natural graphite particles act as crack nails, thus improving the mechanical and thermal properties of BFRP. 
It can be seen that the performance of BFRP materials is achieved mainly by treating the fiber surface and adding admixtures to the substrate. The working mechanism is as follows. (1) By treating the fiber surface, the bond strength between the fiber and the substrate is increased, and the sliding damage is reduced, so that most fibers show "pulloff" damage. When the fiber could reach the yield strength, the composite will be damaged. Thus, the fiber utilization rate is improved and the mechanical properties of the composite are improved. (2) By adding some tiny particles into the epoxy resin substrate, the microstructure of the substrate is affected in some way. The defects such as small voids in the substrate are reduced, and the stress concentration is reduced. Therefore, the mechanical properties of the substrate are improved, so as to enhance the mechanical properties of the composite.

Second, the properties of BFRP components are studied. It mainly refers to research on the mechanical properties of BFRP material as reinforcement, plate, and net. For example, Zha et al. [11] applied BFRP as reinforcement to concrete beam members and studied the impact bending performance of BFRP reinforced concrete beams. Experiment results indicated that the impact resistance of BFRP reinforced concrete beams was equivalent to that of ordinary reinforced concrete beams, and BFRP reinforced concrete beams could be applied to beam members instead of steel bars. Zhang et al. [12] used BFRP as reinforcement to strengthen concrete column members. They found that BFRP reinforcement significantly improved the ductility of column members, and the combination of inner and outer rectangular BFRP reinforcement was the best method to improve members' ductility. Rui [13] studied the fatigue bond performance of BFRP reinforcement and RAC by drawing test and revealed the influence of design parameters on bond stiffness, bond strength, and residual bond strength. Kim et al. [14] applied BFRP as grid cloth to the reinforcement and repair of existing concrete members and conducted relevant experiments. The results showed that BFRP grid cloth could effectively delay the failure time of damaged concrete members. Liu [15] applied BFRP as a laminated plate to strengthen prestressed reinforced concrete beams and compared it with the traditional CFRP laminated plate. It was found that BFRP had greater failure strain and strength and showed significant creep fracture behavior. It can be seen that the BFRP component has excellent mechanical properties and enjoys great potentials in the field of existing structural reinforcement engineering.

Based on the above analysis, scholars at home and abroad have extensively studied the properties of BFRP materials and components and obtained fruitful achievements, which played a positive role in promoting the development of BFRP materials. The existing studies are largely based on the modification of fiber surface and substrate microstructure to improve the properties of the composite. However, there is little research on the impact of fiber distribution on the mechanical properties of BFRP. In practice, due to the different molding processes of BFRP, it is difficult to achieve uniform distribution of fibers in the matrix material, and fiber agglomeration may occur [16-18].
Therefore, based on the "fiber clustering model" proposed in [19], this paper analyzes the geometric shape of the cluster fiber section on the fracture surface of BFRP materials by using the fractal theory and obtains the actual fiber uniform distribution coefficient and the clustering fiber content of BFRP materials. Besides, this paper compares the above values with those obtained from the approximate calculation method and proposes a composite tensile strength model considering fiber clustering effect based on fractal theory. It provides theoretical support for the strength prediction of fiber/epoxy resin composites.

\section{Tensile Fractal Model of Composite Materials considering Fiber Clustering Effect}

2.1. Fractal Geometric Characteristics of Cluster Fibers. This section analyzes the geometric characteristics of one fiber agglomerate. In Figure 1, the black part is the true cross-sectional area of the fiber agglomerate. Figure 2 shows its internal void area, and Figure 3 presents the cross-sectional perimeter. To analyze the fiber beating effect based on the "fiber beating model" proposed in [19], it is necessary to know the indexes such as fiber uniform distribution coefficient and fiber beating content. In this case, this paper needs to obtain the important parameters for calculating the fiber nominal distribution coefficient and fiber beating content, including the true cross-sectional area, nominal cross-sectional area (the sum of the true cross-sectional area and the internal void area of the fiber agglomerate), and the cross-sectional perimeter of the fiber agglomerate. In practice, the cross section of fiber agglomerates is composed of irregular circular bodies arranged randomly, so it is difficult to calculate the real area of fiber agglomerates by Euclidean geometry theory. Reference [19] assumes that fibers in the agglomerate are arranged in squares and triangles, and the fiber uniform distribution coefficient and fiber cluster content are calculated by the approximate method, which is different from the reality. Therefore, how to calculate the true cross-sectional area and the true crosssectional perimeter of fiber agglomerates is the key to analyzing the fiber clustering effect.

In this case, this paper analyzes the cross-sectional features of real fiber agglomerates. They can be considered to be formed by arranging fibers according to certain physical rules (as shown in Figure 1), and their cross-sectional perimeters are formed by arcs of different lengths (as shown in Figure 3). It can be seen that the cross-sectional area of each fiber agglomerate is similar in shape, and the cross-sectional perimeter is self-similar in shape. According to the fractal theory [20-22], the set of shapes with self-similarity has fractal features. Therefore, the cross-sectional area and crosssectional perimeter of fiber agglomerates have fractal characteristics.

\subsection{Analysis of Fiber Clustering Effect Based on Fractal Theory.} In this section, the relationship between fiber uniform distribution coefficient and fractal dimension of fiber agglomerates is analyzed by using relevant principles of fractal 


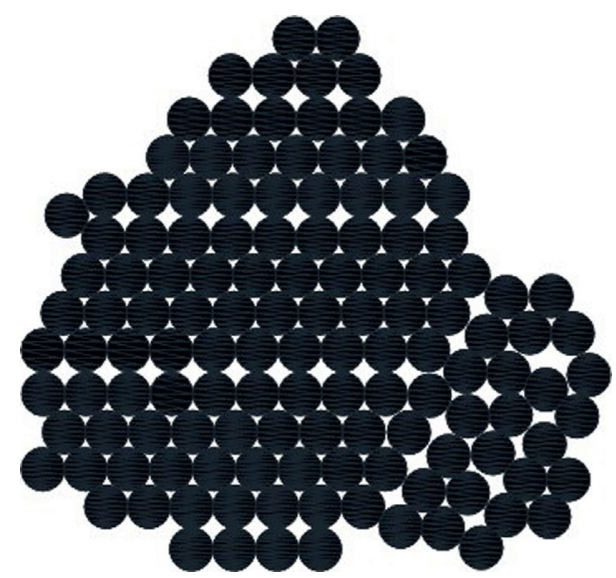

Figure 1: Cross-sectional area of the cluster fiber.

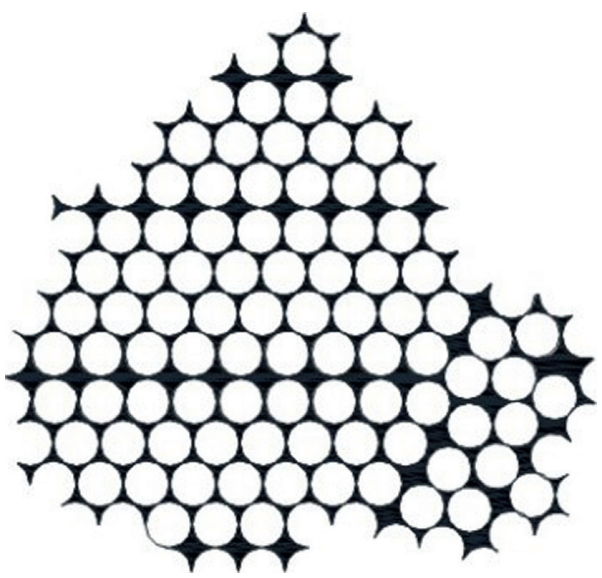

FIGURE 2: Internal void area of the cluster fiber.

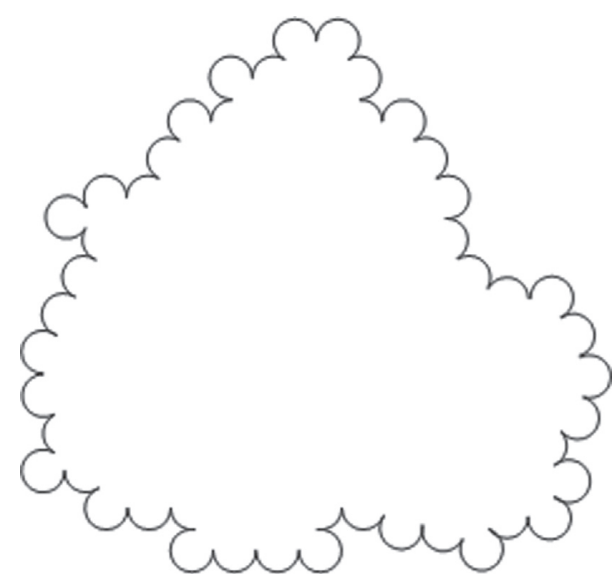

Figure 3: Section perimeter of the cluster fiber.

theory. The box counting dimension method [23] is used to calculate the cross-sectional perimeter $\mathrm{C}$ and cross-sectional area $S$ of the fiber agglomerate, as shown in the following equations:

$$
\begin{gathered}
C=M_{1}\left(\delta_{L}\right) \delta_{L}^{D_{L}}, \\
S=M_{2}\left(\delta_{S}\right) \delta_{S}^{D_{S}},
\end{gathered}
$$

where $\delta_{L}$ and $\delta_{S}$ are length and area measurement yardsticks, respectively. According to the box counting method, the circumference of the cross section of the fiber agglomerate contains $M_{1}\left(\delta_{L}\right) \delta_{L}\left(M_{1}\left(\delta_{L}\right)\right.$ is the number of $\left.\delta_{L}\right)$, and the cross section contains $M_{2}\left(\delta_{S}\right) \delta_{S}\left(M_{2}\left(\delta_{S}\right)\right.$ is the number of $\left.\delta_{S}\right) ; D_{L}$ and $D_{S}$ represent the fractal dimensions of the circumference and area of the section of the fiber agglomerate. According to the fiber uniform distribution coefficient defined in [19], the number $(N)$ of fibers contained in the fiber agglomerate can be calculated according to equation (3), and the fiber uniform distribution coefficient can be calculated according to equation (4):

$$
\begin{aligned}
N & =\frac{4 S}{\pi d_{f}^{2}} \\
& =\frac{4 M_{2}\left(\delta_{S}\right) \delta_{S}^{D_{S}}}{\pi d_{f}^{2}}, \\
\eta_{e} & =\frac{\tau l_{f} d_{f}+\mu \sigma_{e} l_{f} d_{f}}{8 \tau l_{f}} \cdot \frac{M_{1}\left(\delta_{L}\right) \delta_{L}^{D_{L}}}{M_{2}\left(\delta_{S}\right) \delta_{S}^{D_{S} D_{S}}},
\end{aligned}
$$

where $\tau$ is the bonding strength between fibers and the substrate, $\mu$ indicates the friction coefficient between fibers and the substrate, $d_{f}$ is the diameter of fibers, $l_{f}$ is the length of fibers, and $\sigma_{e}$ represents the shrinkage stress of the substrate. When calculating the circumference and area of a fiber agglomerate by box counting method, it is assumed that the side length of its square box is $\delta_{0}$. Then,

$$
\begin{aligned}
& \delta_{L}=\delta_{0}, \\
& \delta_{S}=\delta_{0}^{2} .
\end{aligned}
$$

Substitute equations (5) and (6) into (4), and the following formula can be obtained:

$$
\eta_{e}=\frac{\tau l_{f} d_{f}+\mu \sigma_{e} l_{f} d_{f}}{8 \tau l_{f}} \cdot \frac{M_{1}\left(\delta_{0}\right)}{M_{2}\left(\delta_{0}^{2}\right)} \cdot \delta_{0}^{D_{L}-2 D_{s}} .
$$

When fibers and the substrate are determined, $\tau, \mu, d_{f}$, $l_{f}$, and $\sigma_{e}$ are all constants. For a certain fiber agglomerate, $M_{1}\left(\delta_{L}\right)$ and $M_{2}\left(\delta_{S}\right)$ are also constants. Therefore, let

$$
\frac{\tau l_{f} d_{f}+\mu \sigma_{e} l_{f} d_{f}}{8 \tau l_{f}}=u \text {. }
$$

Then, equation (7) can be simplified as

$$
\eta_{e}=u \cdot \frac{M_{1}\left(\delta_{0}\right)}{M_{2}\left(\delta_{0}^{2}\right)} \cdot \delta_{0}^{D_{L}-2 D_{S}} .
$$

Equation (9) is the fractal expression of the uniform distribution coefficient of the $i^{\text {th }}$ fiber agglomerate. If the composite contains $j$ fiber agglomerates, the fiber uniform 
distribution coefficient of the composite is the average value of $j$ fiber agglomerates. As shown in equation (10), the fiber clustering content $w$ can be calculated according to equation (11).

$$
\begin{aligned}
\eta_{e j} & =\frac{u}{j} \cdot \sum_{i=1}^{j}\left[\frac{M_{1}\left(\delta_{j 0}\right)}{M_{2}\left(\delta_{j^{0}}^{2}\right)} \cdot \delta_{j 0}^{D_{L}-2 D_{S}}\right], \\
w & =\frac{V_{e}}{V_{f}} \\
& =\frac{l_{f} \cdot \sum_{i=1}^{j} M_{2}\left(\delta_{j^{0}}^{2}\right) \cdot \delta_{j 0}^{2 D_{S}}}{V_{f}},
\end{aligned}
$$

where $V_{e}$ is the volume fraction of agglomerated fiber and $V_{f}$ is the fiber volume fraction.

\subsection{Fractal Prediction Model of Tensile Strength of Composite} Materials considering Fiber Clustering Effect. According to the composite mechanics theory [24, 25], FRP is regarded as a multi-item composite elastic system with fiber reinforced resin. The strength and elastic modulus of the composite are equal to the elastic superposition of the properties of each component. The mechanical model is shown in Figure 4.

Therefore, the stress relationship in BFRP elastic stage can be expressed as

$$
\begin{aligned}
\sigma_{f c} & =\sigma_{f} V_{f}+\sigma_{m} V_{m} \\
& =\sigma_{m}\left[1+(n-1) V_{f}\right],
\end{aligned}
$$

where $n=E_{f} / E_{m}, V_{m}+V_{f}=1, E_{f c}$ indicates elastic modulus of FRP, and $\sigma_{f c}$ is the stress of FRP in elastic stage; $\sigma_{m}$ represents the stress of epoxy resin matrix and $\sigma_{f}$ is the stress of fiber; $V_{m}$ is the volume ratio of epoxy resin. It can be seen that the tensile initial cracking strength of FRP depends on the tensile strength of epoxy resin. The larger the elastic modulus ratio of fiber to epoxy resin, the greater the initial cracking strength of FRP. Assuming that epoxy resin cracks after reaching its tensile strength under tensile load, and all load is transferred to the fiber until it is broken, then

$$
\sigma_{f c}^{u}=\eta_{f} \sigma_{f}^{u} V_{f}
$$

where $\sigma_{f c}^{u}$ is FRC tensile strength, $\sigma_{f}^{u}$ is the fiber tensile strength, and $\eta_{f}$ is the fiber effective coefficient. Then, the FRC tensile strength considering fiber clustering effect is expressed as

$$
\sigma_{f c}^{u}=\eta_{f} \sigma_{f}^{u} V_{f}(1-w)+\eta_{e} \eta_{f} \sigma_{f}^{u} V_{f} w
$$

Substitute equations (11) and (12) into (14), and the FRP fractal analytical equation of ultimate tensile strength can be obtained:

$$
\sigma_{f c}^{u}=\eta_{f} \sigma_{f}^{u}\left(V_{f}-l_{f} \cdot \sum_{i=1}^{j} M_{2}\left(\delta_{j 0}^{2}\right) \cdot \delta_{j 0}^{2 D_{s}}\right)+\frac{u}{j} \cdot \sum_{i=1}^{j}\left[\frac{M_{1}\left(\delta_{j 0}\right)}{M_{2}\left(\delta_{j 0}^{2}\right)} \cdot \delta_{j 0}^{D_{L}-2 D_{S}}\right] \cdot \eta_{f} \sigma_{f}^{u} l_{f} \cdot \sum_{i=1}^{j} M_{2}\left(\delta_{j 0}^{2}\right) \cdot \delta_{j 0}^{2 D_{S}} .
$$

According to equation (15), if the fiber orientation, length, and volume fraction are fixed, the FRP tensile strength under fiber clustering effect is only related to the fractal dimension of the fiber agglomerate's perimeter and area.

\section{Tensile Properties Analysis of BFRP Based on Fractal Theory}

3.1. Fractal Geometric Characteristics of Fiber Agglomerates in BFRP Tensile Specimens. Taking BFRP materials prepared in [19] as an example, this article selects the BFRP samples with fiber volume fraction of $0.6 \%, 0.9 \%, 1.2 \%$, and $1.5 \%$ to represent the cross section. After cutting and grinding, they are observed at 500 times magnification, and the arrangement of fiber agglomerate number and internal fibers under different fiber volume fractions is obtained. For example, Figure 5 shows the gray-scale image of a fiber agglomerate when the volume fraction is $1.2 \%$. The grayscale image is binarized (an image containing only black and white colors). That is, in the gray-scale image, there are significant differences in gray-scale values among fibers, base materials, and fiber agglomerates. The grayscale values of cluster fiber cross section are taken as the threshold. Then, the regions with gray-scale values larger than the threshold are all white by default; otherwise, they are black, thus obtaining the cross-sectional arrangement and distribution of clustered fibers. As shown in Figure 6, the white part is the fiber cross section, and the black part is the gap between base materials and fiber clusters. Taking Figure 6 as an $m \times n$ matrix digital image with a height of $m$ and a width of $n$, different pixel images can be obtained by changing the values of $m$ and $n$. Therefore, there is oneto-one correspondence between pixel positions in the image and element positions in the matrix. Since the binarized image only contains two colors (black and white), the elements of $m \times n$ matrix can be expressed by two numbers. Here, the element value " 1 " represents black color and " 0 " represents white color.

The fractal dimension of the area of white part in Figure 6 is calculated by box counting method. The principle is that square boxes with different area sizes $\left(\delta_{S i}\right)$ are used to cover the images of the white area. At the same time, the number of boxes contained in the cross-sectional area of each fiber agglomerate $M_{2 i}\left(\delta_{S}\right)$ was recorded. Then, $M_{2}\left(\delta_{S}\right)$ can be expressed as

$$
M_{2 i}\left(\delta_{S i}\right)=a \delta_{S i}^{-D_{S}}
$$

where $a$ is a constant. Then, take the logarithm of equation (16): 


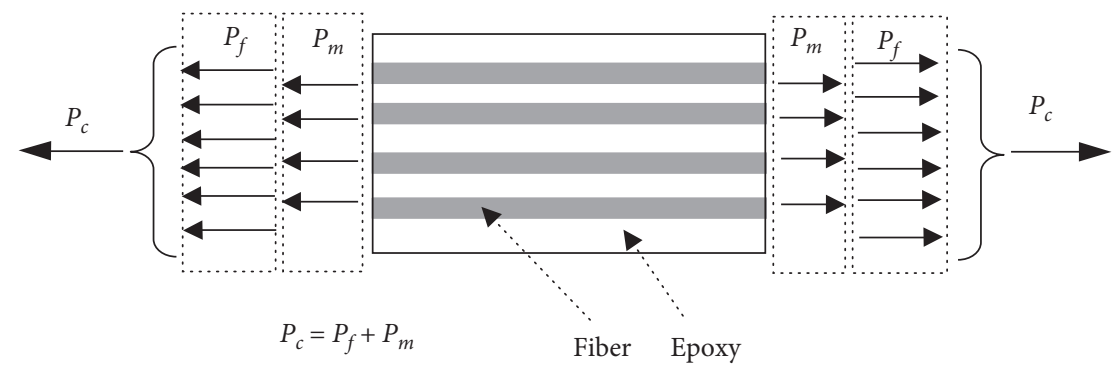

FIgURE 4: Mechanical model of BFRP composite materials.

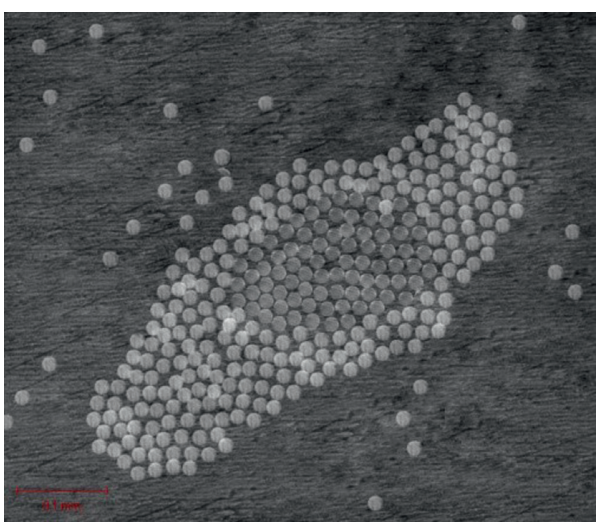

Figure 5: The cluster fiber.

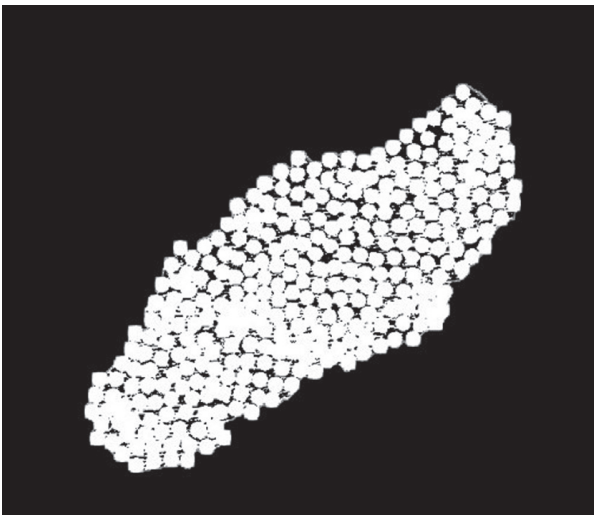

Figure 6: The effect of cluster fiber with binary treatment.

$$
\ln M_{2 i}\left(\delta_{S i}\right)=\ln a-D_{S} \ln \delta_{S i}
$$

According to equation (17), the fractal dimension of the cross-sectional area of the clustering fiber, $D_{S}$, can be obtained from the linear regression analysis on the set (ln $\left.M_{2}\left(\delta_{S}\right), \ln \delta_{S i}\right)$ generated by the box counting method. It is the absolute value of the slope of the curve.

Divide the gray image of the fiber agglomerate in Figure 6 to obtain the contour map of the fiber agglomerate section, as shown in Figure 7. Likewise, the fractal dimension of contour line in Figure 7 is calculated by the box counting method. Then, the fractal dimension of fiber agglomerate cross-sectional perimeter is obtained. Record the number of

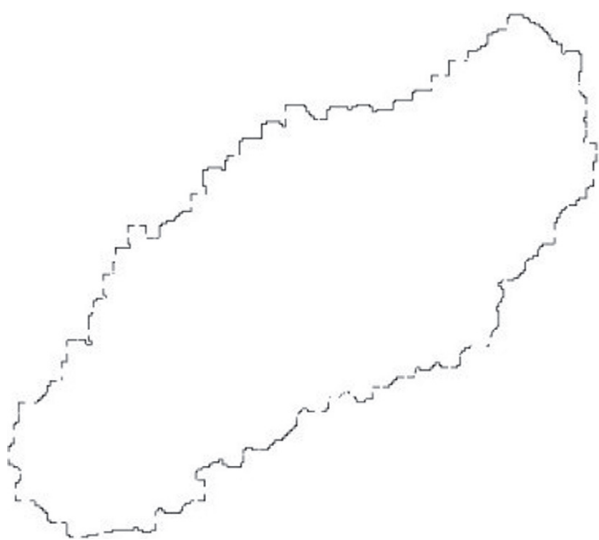

FIgURE 7: The outline drawing of cluster fibers.

boxes included in the contour line image of the fiber agglomerate cross section as $M_{1 i}\left(\delta_{L}\right)$; then

$$
\lg M_{1 i}\left(\delta_{L i}\right)=\lg b-D_{L} \lg \delta_{L i},
$$

where $b$ is a constant. Then, the set $\left(\lg M 1\left(\delta_{L}\right), \lg \delta L i\right)$ is generated by the box counting method, and the method of calculating $D_{S}$ is utilized to calculate $D_{L}$. The data points with self-similar characteristics in a limited scale range are fitted, and the slope of the obtained curve is the fractal dimension $D_{L}$ of the cross-sectional profile of the cluster fiber (cross-sectional perimeter of the cluster fiber).

Based on the above methods, the fractal dimension of fiber agglomerate cross-sectional area and perimeter on the fracture surface of BFRP samples with different fiber volume doping rates can be obtained. As shown in Figure 8, the fractal dimension of fiber agglomerate cross-sectional area is about 1.8, and the fractal dimension of cluster fiber crosssectional perimeter is about 1.1 .

Take the square box area with a side length of $0.01 d_{f}$ as the benchmark, that is, $\delta_{S}=\left(0.01 d_{f}\right)^{2}$, and record the number of boxes, $M_{2}\left(\delta_{S}\right)$. The area of the white part, $S_{f}$, can be obtained through equation (2). Besides, start measuring with $\delta_{L}=0.01 d_{f}$ as the side length of the box and record the number of boxes $M_{1}\left(\delta_{L}\right)$. Then, the perimeter $C_{f}$ of different fiber agglomerate cross-sectional contours can be obtained through equation (1). Table 1 shows the crosssectional areas and circumferences of fiber clusters on fracture surfaces of different BFRP samples calculated based on fractal theory. From this table, it can be seen that the 


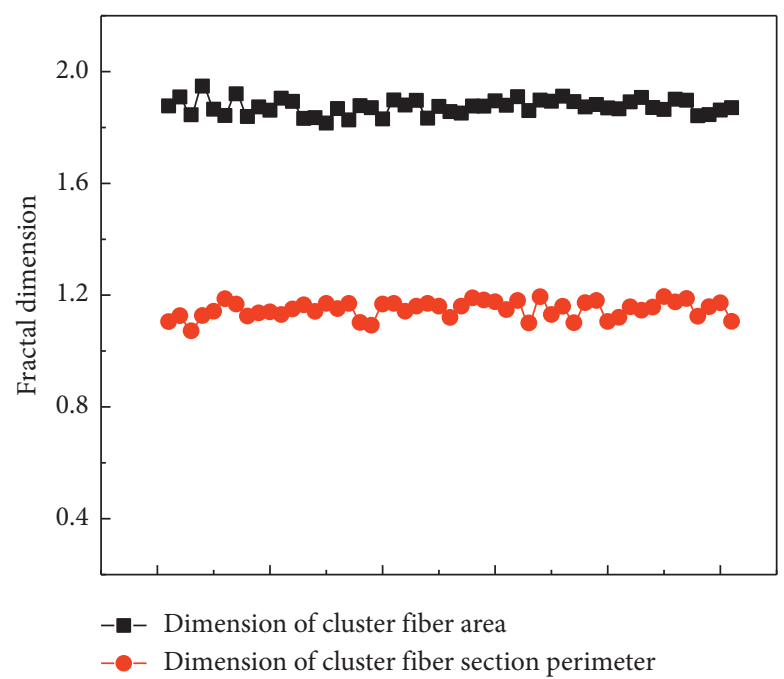

Figure 8: The fractal dimensions of cluster fiber section area and section perimeter.

TABle 1: Calculated values of $c_{j}$ and $S_{j}$ with fractal method.

\begin{tabular}{|c|c|c|c|c|c|c|c|c|}
\hline \multirow[b]{2}{*}{$j$} & \multicolumn{2}{|c|}{$i=0.6 \%$} & \multicolumn{2}{|c|}{$i=0.9 \%$} & \multicolumn{2}{|c|}{$i=1.2 \%$} & \multicolumn{2}{|c|}{$i=1.5 \%$} \\
\hline & $c_{j}(\mathrm{~mm})$ & $S_{j}\left(\mathrm{~mm}^{2}\right)$ & $c_{j}(\mathrm{~mm})$ & $S_{j}\left(\mathrm{~mm}^{2}\right)$ & $c_{j}(\mathrm{~mm})$ & $S_{j}\left(\mathrm{~mm}^{2}\right)$ & $c_{j}(\mathrm{~mm})$ & $S_{j}\left(\mathrm{~mm}^{2}\right)$ \\
\hline 1 & 1.7432 & 0.1027 & 2.3216 & 0.1995 & 1.8216 & 0.0817 & 2.0516 & 0.0995 \\
\hline 2 & 2.1893 & 0.1763 & 2.1325 & 0.0838 & 1.5367 & 0.0841 & 1.4216 & 0.1071 \\
\hline 3 & 1.2563 & 0.0434 & 1.6824 & 0.0788 & 1.9421 & 0.1146 & 1.4452 & 0.1132 \\
\hline 4 & 0.9864 & 0.0391 & 1.9387 & 0.0763 & 1.2673 & 0.1171 & 1.6457 & 0.0906 \\
\hline 5 & 1.9322 & 0.078 & 1.8632 & 0.1363 & 1.4683 & 0.0934 & 1.7683 & 0.1074 \\
\hline 6 & 1.8861 & 0.0703 & 1.8914 & 0.139 & 1.5214 & 0.0665 & 1.4519 & 0.106 \\
\hline 7 & - & - & 1.7325 & 0.0815 & 1.7658 & 0.0961 & 1.7218 & 0.1064 \\
\hline 8 & - & - & 1.8106 & 0.0652 & 1.5416 & 0.0888 & 1.6457 & 0.0698 \\
\hline 9 & - & - & 1.4563 & 0.0524 & 1.5317 & 0.0697 & 1.7549 & 0.0954 \\
\hline 10 & - & - & - & - & 1.2549 & 0.1022 & 1.4318 & 0.0895 \\
\hline 11 & - & - & - & - & 1.2463 & 0.1162 & 1.5673 & 0.1316 \\
\hline 12 & - & - & - & - & 1.6715 & 0.116 & 1.4983 & 0.0762 \\
\hline 13 & - & - & - & - & 1.6124 & 0.1275 & 1.8473 & 0.0846 \\
\hline 14 & - & - & - & - & - & - & 1.3981 & 0.0893 \\
\hline 15 & - & - & - & - & - & - & 1.5428 & 0.0907 \\
\hline 16 & - & - & - & - & - & - & 1.4672 & 0.0887 \\
\hline 17 & - & - & - & - & - & - & 1.6427 & 0.0574 \\
\hline 18 & - & - & - & - & - & - & 1.946 & 0.0801 \\
\hline 19 & - & - & - & - & - & - & 1.917 & 0.0944 \\
\hline
\end{tabular}

circumference of fiber agglomerate section is about $0.9-2.3 \mathrm{~mm}$. Compared with the results of the approximate method, the circumference of fiber agglomerate section based on fractal theory has increased. It is due to the fact that different benchmarks can be used for actual measurement when calculating by the box counting method, and the smaller the scale, the longer the circumference of the fiber agglomerate. Therefore, the perimeter calculated by fractal theory is accurate in strict sense. The cross-sectional area of the fiber agglomerate calculated by this method is about $0.03-0.17 \mathrm{~mm}^{2}$, which is smaller than that calculated by the approximate method. This is because the calculated area of fractal theory is based on the binarization result of the enlarged image of the fiber cluster, which reflects the true arrangement of fibers in the agglomerate. Therefore, the calculated results are more objective.

3.2. Analysis of BFRP Fiber Clustering Effect Based on Fractal Theory. After substituting the data in Table 1 into equations (8) and (10), the value of fiber uniform distribution coefficient of BFRP samples based on fractal theory can be obtained. Combined with the results of [19] (calculated by approximate method), it is found that the results based on fractal theory have similar rules with the calculated results of [19]: (1) the fiber uniform distribution coefficient decreases with the increase of fiber volume fraction; (2) if the fiber volume fraction is constant, the fiber uniform distribution 


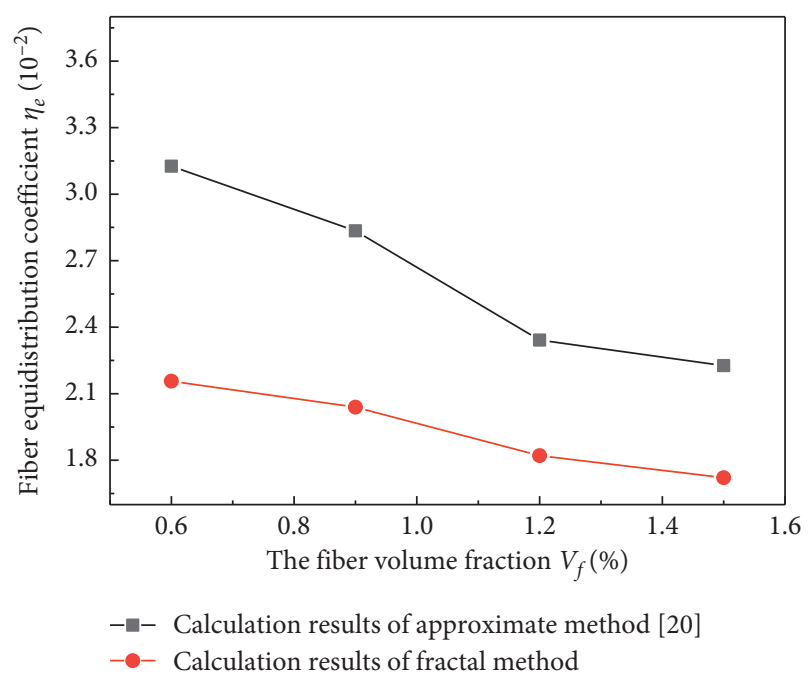

Figure 9: Fiber equidistribution coefficient as a function of fiber volume fraction.

coefficients calculated by fractal theory are all lower than those calculated by the approximate method, as shown in Figure 9.

Substitute the data in Table 1 into equation (11), and the value of fiber clustering content of BFRP samples based on fractal theory can be obtained. Combining the above value with the results of [19] (calculated by approximate method), it can be found that the fiber clustering content increases with the increase of fiber volume fraction. Comparing the above value with the calculated results by the approximate method, it can be concluded that when the fiber volume fraction is constant, the calculated value of fiber clustering content by fractal theory is higher than that by the approximate method. As shown in Figure 10, when the fiber volume fraction is greater than $1.2 \%$, the increase of the calculated values under fractal theory has slowed down.

\subsection{Tensile Strength Analysis of BFRP Based on Fractal Theory.} Based on the analysis in Section 3.2, this paper substitutes the calculated values of $M_{1}\left(\delta_{L}\right), M_{2}\left(\delta_{S}\right), c_{j}$, and $S_{j}$ into equation (15) and obtains the composite tensile strength based on fractal theory. Then, the results are compared with those of [19], as shown in Figure 11.

It can be seen that, with the increase of fiber volume fraction, the calculated value of composite tensile strength displays a similar trend with the experimental value. When the fiber volume fraction is constant, the calculated value of composite tensile strength under fractal theory is slightly lower than the experimental value. Through the regression analysis between the results obtained by the two methods and the experimental values, it is found that the calculated values of BFRP tensile strength based on fractal theory are in good agreement with the experimental values, and the correlation coefficient is as high as 0.941 , which is closer to the experimental results than the calculated results of [19] (approximate method). It can provide theoretical basis for the design and strength prediction of BFRP composites.

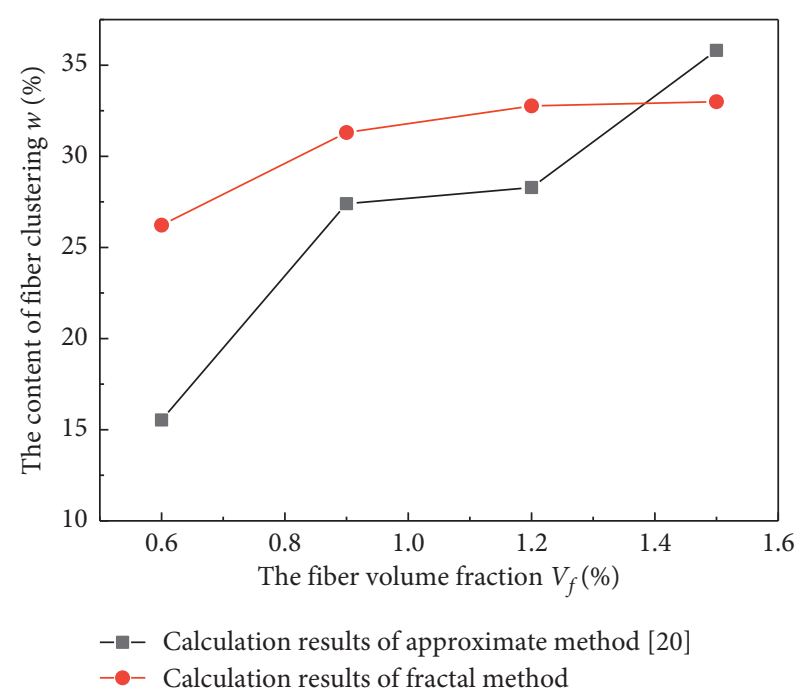

Figure 10: The trend of content of fiber clustering with fiber volume fraction by fractal method.

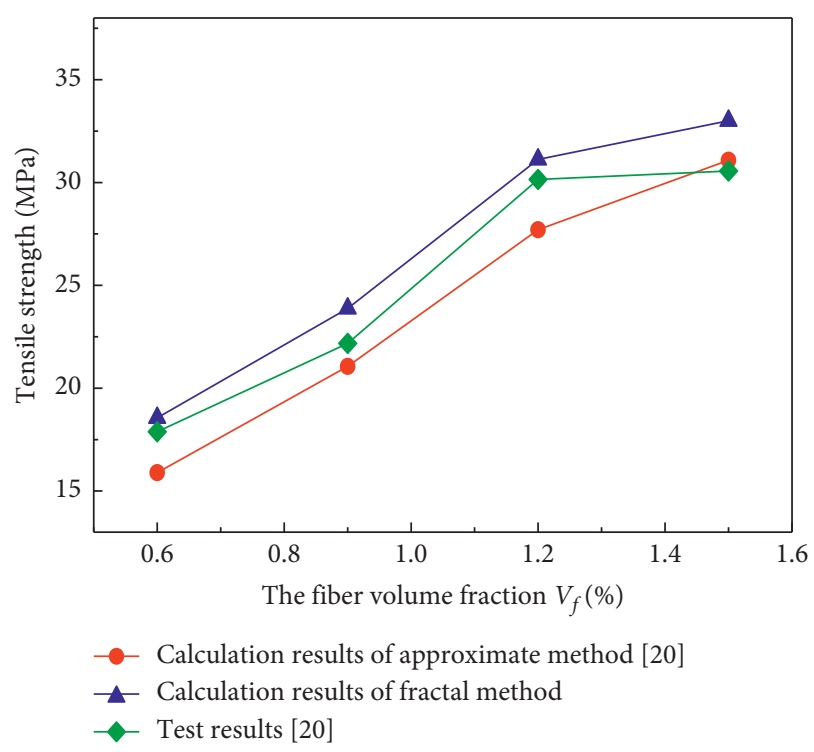

Figure 11: The calculated values of composite materials tensile strength with different methods and test values.

\section{Conclusion}

In this paper, the fractal characteristics of agglomerated fibers were analyzed and established a prediction model of tensile strength of fiber/epoxy resin composites containing agglomerated fibers. With BFRP materials as an example, the following conclusions were reached:

(1) When the fiber orientation, length, and volume fraction are fixed, the tensile strength of the composite containing agglomerated fibers is only related to the fractal dimension of the circumference and area of the inner fiber agglomerate.

(2) The fractal dimensions of cross-sectional area and perimeter of fiber agglomerates in BFRP are about 
1.8 and 1.1, respectively. The calculated values of fiber uniform distribution coefficient and fiber clustering content based on fractal theory are similar to those calculated in [19]. It means the fiber uniform distribution coefficient decreases with the increase of fiber volume fraction, while the fiber clustering content increases with the increase of fiber volume fraction. The difference is that the fiber uniform distribution coefficient calculated by fractal theory is lower than that calculated by the approximate method, and the fiber clustering content calculated by fractal theory is higher than that calculated by the approximate method.

(3) The calculated value of BFRP tensile strength based on fractal theory is lower than the experimental value, but closer to the experimental value than the calculated value of the approximate method. The research conclusion can provide theoretical support for BFRP strength prediction.

\section{Data Availability}

Some or all data, models, or code generated or used during the study are available from the corresponding author upon request.

\section{Conflicts of Interest}

The authors declare that they have no conflicts of interest.

\section{Acknowledgments}

The authors gratefully acknowledge the support of the Natural Science Basic Research Program of Shaanxi China (program no. 2021JQ-983).

\section{References}

[1] J. H. Lee, K. Y. Rhee, and S. J. Park, "The tensile and thermal properties of modified cnt-reinforced basalt/epoxy composites," Materials Science and Engineering, vol. 527, no. 26, pp. 6838-6843, 2010.

[2] M. R. Ricciardi, I. Papa, G. Coppola, V. Lapresto, L. Sansone, and V. Antonucci, "Effect of plasma surface treatment on the impact behavior of basalt/epoxy composites," Key Engineering Materials, vol. 813, pp. 441-446, 2019.

[3] K. S. Hwang and P. Soo-Jin, "Effect of graphene oxide on interfacial interactions and fracture toughness of basalt fiberreinforced epoxy composites," Journal of Nanoscience and Nanotechnology, vol. 20, no. 11, pp. 6760-6767, 2020.

[4] H. Ulus, H. B. Kaybal, V. Eskizeybek, and A. Avc1, "Halloysite nanotube reinforcement endows ameliorated fracture resistance of seawater aged basalt/epoxy composites," Journal of Composite Materials, vol. 54, no. 20, pp. 2761-2779, 2020.

[5] M. M. Shokrieh and M. Memar, "Stress corrosion cracking of basalt/epoxy composites under bending loading," Applied Composite Materials, vol. 17, no. 2, pp. 121-135, 2010.

[6] M. M. Shokrieh and M. Memar, "Characterization of tensile and compressive properties of basalt/epoxy composites under stress corrossion conditions," Mechanics of Composite $\mathrm{Ma}$ terials, vol. 53, no. 6, pp. 759-766, 2018.
[7] M. Bulut, A. Erkliğ, and P. Kanmaz, "Vibration-damping characterization of the basalt/epoxy composite laminates containing graphene nanopellets," Science and Engineering of Composite Materials, vol. 26, no. 1, pp. 147-153, 2018.

[8] A. Chelliah, "Mechanical properties and abrasive wear of different weight percentage of $\mathrm{TiC}$ filled basalt fabric reinforced epoxy composites," Materials Research, vol. 22, no. 2, 2019.

[9] P. Sampath and S. Santhanam, "Effect of moringa and bagasse ash filler particles on basalt/epoxy composites," Polímeros-Ciência e Tecnologia, vol. 29, no. 3, 2019.

[10] S. H. Kim, Y.-J. Heo, M. Park, B.-G. Min, K. Y. Rhee, and S.-J. Park, "Effect of hydrophilic graphite flake on thermal conductivity and fracture toughness of basalt fibers/epoxy composites," Composites Part B: Engineering, vol. 153, pp. 9-16, 2018.

[11] Z. J. Huang, W. S. Chen, T. T. Tran et al., "Experimental and numerical study on concrete beams reinforced with basalt FRP bars under static and impact loads," Composite Structures, vol. 263, Article ID 113648, 2021.

[12] P. Zhang, B. Chen, Y. Liu et al., "Experimental study on the axial compression behavior of columns confined by BFRP ties," Composite Structures, vol. 258, Article ID 113302, 2021.

[13] R. Zhou, F. Liu, Z. Xiong, S. H. He, L. J. Li, and W. Wei, "Experimental study on fatigue bond behaviour between basalt fibre-reinforced polymer bars and recycled aggregate concrete," Construction and Building Materials, vol. 270, Article ID 121399, 2021.

[14] Y. J. Kim and M. Solanki, "Post-Peak crack control of concrete with BFRP grids," Construction and Building Materials, vol. 282, Article ID 122716, 2021.

[15] C. Liu, X. Wang, J. Shi, L. Liu, and Z. Wu, "Experimental study on the flexural behavior of RC beams strengthened with prestressed BFRP laminates," Engineering Structures, vol. 233, no. 4, Article ID 111801, 2021.

[16] J. Zhou, S. Qian, G. Ye, O. Copuroglu, K. Van Breugel, and V. C. Li, "Improved fiber distribution and mechanical properties of engineered cementitious composites by adjusting the mixing sequence," Cement and Concrete Composites, vol. 34, no. 3, pp. 342-348, 2012.

[17] C. Yang, H.-X. Huang, and K. Li, "Investigation of fiber orientation states in injection-compression molded short-fiber-reinforced thermoplastics," Polymer Composites, vol. 31, no. 11, pp. 1899-1908, 2010.

[18] X. J. Tan and Q. S. Yang, "Influence of microstructure on effective properties of fiber bundle reinforced composites," Acta Materiae Compositae Sinica, vol. 26, no. 3, pp. 188-194, 2009, in Chinese.

[19] J. J. He, J. P. Shi, X. S. Cao, and Y. F. Hu, "Tensile mechanical properties and failure modes of a basalt fiber/epoxy resin composite material," Advances in Civil Engineering, vol. 2018, Article ID 7914727, 10 pages, 2018.

[20] B. B. Mandelbrot, D. E. Passoja, and A. J. Paullay, "Fractal character of fracture surfaces of metals," Nature, vol. 26, no. 308, pp. 721-722, 1984.

[21] O. Hilders and N. Zambrano, "The effect of aging on impact toughness and fracture surface fractal dimension in SAF 2507 super duplex stainless steel," Journal of Microscopy and Ultrastructure, vol. 2, no. 4, pp. 236-244, 2014.

[22] T. B. Mcmurphy, C. A. Harris, and J. A. Griggs, "Accuracy and precision of fractal dimension measured on model surfaces," Dental Materials, vol. 30, no. 3, pp. 302-307, 2014.

[23] S. B. Chen, X. W. Pan, and J. F. Liu, "Impact localization method based on the partial least squares regression fractal 
dimension," Journal of Vibration and Shock, vol. 40, no. 2, pp. 98-102, 2021, in Chinese.

[24] S. W. Tsai and E. M. Wu, "A general theory of strength for anisotropic materials," Journal of Composite Materials, vol. 5, no. 1 , pp. 58-80, 1971.

[25] K. S. Liu and S. W. Tsai, "A progressive quadratic failure criterion for a laminate 1," Composites Science and Technology, vol. 58, no. 7, pp. 1023-1032, 1998. 